\title{
ELLIPTIC SOLUTIONS TO NONSYMMETRIC MONGE-AMPÈRE TYPE EQUATIONS I. THE $d$-CONCAVITY AND THE COMPARISON PRINCIPLE
}

\author{
HA TIEN NGOAN AND THAI THI KIM CHUNG
}

\begin{abstract}
We introduce the so-called $d$-concavity, $d \geq 0$, and prove that the nonsymmetric Monge-Ampère type function of matrix variable is concave in an appropriate unbounded and convex set. We prove also the comparison principle for nonsymmetric Monge-Ampère type equations in the case when they are so-called $\delta$-elliptic with respect to compared functions with $0 \leq \delta<1$.
\end{abstract}

\section{INTRODUCTION}

In this paper we consider the following nonsymmetric Monge-Ampère type equations:

$$
\operatorname{det}\left[D^{2} u-A(x, u, D u)-B(x, u, D u)\right]=f(x, u, D u), x \in \Omega,
$$

where $\Omega$ is a bounded domain in $n$ dimensional Euclidean space $\mathbb{R}^{n}$ with smooth boundary, $D u$ and $D^{2} u$ denote the gradient vector and the Hessian matrix of the second order derivatives of the function $u: \Omega \rightarrow \mathbb{R}$, respectively, $A$ is a given $n \times n$ symmetric matrix function defined on $\Omega \times \mathbb{R} \times \mathbb{R}^{n}, B$ is a given $n \times n$ skew-symmetric matrix function defined on $\Omega \times \mathbb{R} \times \mathbb{R}^{n}, f$ is a positive scalar valued function defined on $\Omega \times \mathbb{R} \times \mathbb{R}^{n}$. As usual, we use $x, z, p, r$ to denote points in $\Omega, \mathbb{R}, \mathbb{R}^{n}, \mathbb{R}^{n \times n}$, respectively.

In the case that $B(x, z, p) \equiv 0$, equation (1.1) becomes

$$
\operatorname{det}\left[D^{2} u-A(x, u, D u)\right]=f(x, u, D u), x \in \Omega .
$$

For functions $u(x) \in C^{2}(\Omega)$, we set

$$
\omega(x, u) \equiv D^{2} u(x)-A(x, u(x), D u(x)) .
$$

We recall that the equation (1.1) or (1.2) is elliptic with respect to function $u(x) \in$ $C^{2}(\Omega)$ whenever

$$
\lambda_{\min }(\omega(x, u))>0, \forall x \in \Omega
$$

2010 Mathematics Subject Classification. 35J66.

Key words and phrases. $d$-concavity, $\delta$-elliptic, the comparison principle. 
Here and in what follows, we denote by $\lambda_{\min }(M)$ the smallest eigenvalue of a symmetric matrix $M \in \mathbb{R}^{n \times n}$.

For the Dirichlet problem for equation (1.2), the existence of elliptic solutions was settled in [4, [5], [6] by the method of continuity. In this method, the solvability of the Dirichlet problem is reduced to the establishment of $C^{2, \alpha}(\bar{\Omega})$ estimates for its elliptic solutions. It is well-known that the concavity of the following function

$$
F(\omega)=\log (\operatorname{det} \omega),
$$

considered as a function on the set of symmetric positive definite matrices $\omega=$ $\left[\omega_{i j}\right]_{n \times n}$, has one of essential roles in establishing these a priori estimates.

As it had been remarked in [6], the question on the solvability of Dirichlet problem for equations (1.1) when $B(x, z, p) \not \equiv 0$ is an open one. To investigate this problem, instead of the function $F(\omega)$, we will consider in the below the following function of matrix variable,

$$
F(R)=\log (\operatorname{det} R)
$$

where $R=\left[R_{i j}\right] \in \mathbb{R}^{n \times n}$, which is represented by the form

$$
R=\omega+\beta, \omega^{T}=\omega, \omega>0, \beta^{T}=-\beta .
$$

We will show in the below that $\operatorname{det} \beta \geq 0$ and will prove that

$$
\operatorname{det} R=\operatorname{det}(\omega+\beta) \geq \operatorname{det} \omega+\operatorname{det} \beta>0 .
$$

Thus the matrix $R=\omega+\beta$ is always non-singular, the function $F(R)$ is welldefined and infinitely differentiable. The function $F(R)$ is called the MongeAmpère type function, associated to equation (1.1).

Suppose that $\delta, \mu$ are fixed nonnegative numbers, where $\delta \in[0,1)$. For the function $F(R)$, we consider the following set of matrices:

$D_{\delta, \mu} \equiv\left\{R \mid R=\omega+\beta, \omega^{T}=\omega, \beta^{T}=-\beta, \lambda_{\min }(\omega)>0, \delta \lambda_{\min }(\omega) \geq \mu,\|\beta\| \leq \mu\right\}$.

Here and in what follows, $\|\cdot\|$ denotes the operator norm on $\mathbb{R}^{n \times n}$. It is easy to verify that $D_{\delta, \mu}$ is an unbounded and convex set in $\mathbb{R}^{n \times n}$. If $\delta=0$ then $\mu=0$, $\beta=0$ and the set $D_{0,0}$ consists of symmetric positive definite matrices. In order to generalise the notion of usual concavity for the function $\log (\operatorname{det} \omega)$, we introduce the so-called $d$-concavity for the function $F(R)$.

Definition 1. Suppose that $d \geq 0$ is a nonnegative number. The function $F(R)$ is said to be $d$-concave in the set $D_{\delta, \mu}$ if for any matrices $R^{(0)}=\left[R_{i j}^{(0)}\right]_{n \times n}$ and 
$R^{(1)}=\left[R_{i j}^{(1)}\right]_{n \times n}$ from $D_{\delta, \mu}$, we have

$$
F\left(R^{(1)}\right)-F\left(R^{(0)}\right) \leq \sum_{i, j=1}^{n} \frac{\partial F\left(R^{(0)}\right)}{\partial R_{i j}}\left(R_{i j}^{(1)}-R_{i j}^{(0)}\right)+d .
$$

When $d=0$, the 0 -concavity is indeed the usual concavity. One of our main results in this paper is the Theorem [3, in which we prove that the function $F(R)$ is $d$-concave in the set $D_{\delta, \mu}$ with some $d \geq 0$, which depends only on $\delta$ and $n$.

Another aspect of our studying in this paper is the comparison principle for nonsymmetric Monge-Ampère type equations (1.1). It is well-known that when $B(x, z, p) \equiv 0$, the comparison principle holds for elliptic solutions to the equation (1.2). In [2], this principle had been considered for fully nonlinear second-order elliptic equations. However, in applying to the equation (1.1) to compare functions $u(x), v(x) \in C^{2}(\bar{\Omega})$, the following condition needs to be satisfied: for any $t \in[0,1]$, the matrix $\omega(x,(1-t) u(x)+t v(x))$ must be positive definite for all $x \in \Omega$. But, in general, the equation (1.1) do not satisfy this condition. The new point of this paper is that we can prove in the Theorem 4 the comparison principle to the equation (1.1) in the case when it is $\delta$-elliptic with respect to compared functions.

Definition 2. Suppose that $\delta \in[0,1)$ is a fixed number. We say that the equation (1.1) is $\delta$-elliptic with respect to function $u(x) \in C^{2}(\Omega)$ if it is elliptic with respect to $u(x)$ and

$$
\delta \lambda_{\min }(\omega(x, u)) \geq \mu(B), \forall x \in \Omega,
$$

where $\omega(x, u)$ is defined by (1.3) and

$$
\mu(B) \equiv \sup _{\Omega \times \mathbb{R} \times \mathbb{R}^{n}}\|B(x, z, p)\|,
$$

which is assumed to be finite.

Based on the two results mentioned above, in our incoming paper [3], we will get a priori estimates in $C^{2, \alpha}(\bar{\Omega})$ for $\delta$-elliptic solutions to the Dirichlet problem for (1.1). Moreover, by the method of continuity we will prove in that paper that when $A(x, z, p), f(x, z, p)$ satisfy some conditions which are like those for the Dirichlet problem for (1.2) ([4], [5], 6]), there exists a unique $\delta$-elliptic solution to the Dirichlet problem for (1.1) in the space $C^{2, \alpha}(\bar{\Omega})$ for some $0<\alpha<1$, provided that the matrix $B(x, z, p)$ is sufficiently small.

The structure of the paper is as follows. In Section $\S 2$, we recall the notion of the $2^{\text {nd }}$ compound and its properties for square matrices. In Section $\S 3$, we will study the second differentials for the Monge-Ampère type function $F(R)$ 
in the set $D_{\delta, \mu}$ and prove its $d$-concavity. In the last section, we will prove the comparison principle for the equations (1.1), which are $\delta$-elliptic with respect to compared functions.

\section{The $2^{\text {nd }}$ COMPounds OF SQUARE MATRICES}

Definition 3. ([1]) Let $M=\left[M_{i j}\right]$ be an $n \times n$ matrix with entries in $\mathbb{R}$ or $\mathbb{C}$. Suppose that $i<k$ and $j<\ell$. We denote by $M_{i k, j \ell}^{(2)}$ the minor, which is the determinant at the intersection of rows $i, k$ and columns $j, \ell$ of the matrix $M$, that is,

$$
M_{i k, j \ell}^{(2)}=\left|\begin{array}{ll}
M_{i j} & M_{i \ell} \\
M_{k j} & M_{k \ell}
\end{array}\right| .
$$

When the paires $(i k),(j \ell)$ with $i<k$ and $j<\ell$ are arranged in the lexical order, the resulting $\left(\begin{array}{l}n \\ 2\end{array}\right) \times\left(\begin{array}{l}n \\ 2\end{array}\right)$ matrix, consisting of corresponding minors is called the $2^{\text {nd }}$ compound of the matrix $M$ and written as $M^{(2)}$. In symbols, we write

$$
M^{(2)}=\left[M_{i k, j \ell}^{(2)}\right]_{\left(\begin{array}{c}
n \\
2
\end{array}\right) \times\left(\begin{array}{c}
n \\
2
\end{array}\right)} .
$$

Some principal properties of the $2^{\text {nd }}$ compound matrices are listed in the following proposition.

Proposition 1. ([1]) Let $M$ and $N$ be matrices in $\mathbb{C}^{n \times n}$. Then we have the following assertions:

(i) Binet-Cauchy Theorem:

$$
(M N)^{(2)}=M^{(2)} N^{(2)} .
$$

(ii) $\left(M^{(2)}\right)^{T}=\left(M^{T}\right)^{(2)}$, where $M^{T}$ is the transpose of $M$.

(iii) $\overline{M^{(2)}}=\bar{M}^{(2)}$, where $\bar{M}$ is the complex conjugate of $M$.

(iv) $\left(M^{(2)}\right)^{*}=\left(M^{*}\right)^{(2)}$, where $M^{*}$ is the Hermitian adjoint of $M, M^{*}=\bar{M}^{T}$.

(v) $M$ is non-singular if and only if $M^{(2)}$ is non-singular, and

$$
\left(M^{(2)}\right)^{-1}=\left(M^{-1}\right)^{(2)} \text {. }
$$

(vi) Suppose that $M \in \mathbb{R}^{n \times n}$ and $M$ is symmetric or skew-symmetric, then $M^{(2)}$ is symmetric.

(vii) $(k M)^{(2)}=k^{2} M^{(2)}, \forall k \in \mathbb{C}$.

(viii) If $M=\operatorname{diag}\left(\lambda_{1}, \ldots, \lambda_{n}\right)$, then

$$
M^{(2)}=\operatorname{diag}\left(\lambda_{j} \lambda_{k}, j<k\right) .
$$

To investigate the $d$-concavity of function $F(R)$ in the next section, we need the following proposition. 
Proposition 2. Let $M=\left[M_{i j}\right]$ be a square matrix of order $n$. Then

$$
M^{(2)}+\left(M^{T}\right)^{(2)}=\frac{1}{2}\left(M+M^{T}\right)^{(2)}+\frac{1}{2}\left(M-M^{T}\right)^{(2)} .
$$

Proof. For all $i, j, k, \ell=1, \ldots, n$ such that $i<k, j<\ell$, we have

$$
\begin{aligned}
\left(M+M^{T}\right)_{i k, j \ell}^{(2)} & =\left|\begin{array}{ll}
M_{i j}+M_{j i} & M_{i \ell}+M_{\ell i} \\
M_{k j}+M_{j k} & M_{k \ell}+M_{\ell k}
\end{array}\right| \\
& =2\left(\begin{array}{ll}
M_{i j} & M_{i \ell} \\
M_{k j} & M_{k \ell}
\end{array}|+| \begin{array}{cc}
M_{j i} & M_{\ell i} \\
M_{j k} & M_{\ell k}
\end{array} \mid\right) \\
& -\left(\left|\begin{array}{ll}
M_{i j} & M_{i \ell} \\
M_{k j} & M_{k \ell}
\end{array}\right|+\left|\begin{array}{cc}
M_{j i} & M_{\ell i} \\
M_{j k} & M_{\ell k}
\end{array}\right|-\left|\begin{array}{cc}
M_{i j} & M_{\ell i} \\
M_{k j} & M_{\ell k}
\end{array}\right|-\left|\begin{array}{cc}
M_{j i} & M_{i \ell} \\
M_{j k} & M_{k \ell}
\end{array}\right|\right) \\
& =2\left(\begin{array}{ll}
M_{i j} & M_{i \ell} \\
M_{k j} & M_{k \ell}
\end{array}|+| \begin{array}{ll}
M_{j i} & M_{\ell i} \\
M_{j k} & M_{\ell k}
\end{array} \mid\right)-\left|\begin{array}{ll}
M_{i j}-M_{j i} & M_{i \ell}-M_{\ell i} \\
M_{k j}-M_{j k} & M_{k \ell}-M_{\ell k}
\end{array}\right| \\
& =2\left(M_{i k, j \ell}^{(2)}+\left(M^{T}\right)_{i k, j \ell}^{(2)}\right)-\left(M-M^{T}\right)_{i k, j \ell}^{(2)} .
\end{aligned}
$$

This implies the desired equality (2.1).

3. The $d$-COnCAvity of the nOnsymmetric Monge-Ampère type

\section{FUNCTIONS}

3.1. Some properties of matrices $R$ belonging the set $D_{\delta, \mu}$. Let $D_{\delta, \mu}$ is the set given in (1.5). We shall introduce some properties of matrices $R=\omega+\beta$ from $D_{\delta, \mu}$.

Proposition 3. Suppose that $R=\omega+\beta \in \mathbb{R}^{n \times n}$, where $\omega$ is symmetric positive definite, $\beta$ is skew-symmetric. Then

(i) $\operatorname{det} R \geq \operatorname{det} \omega+\operatorname{det} \beta \geq \operatorname{det} \omega>0$.

(ii) Particularly, when $n=2$,

$$
\operatorname{det} R=\operatorname{det} \omega+\operatorname{det} \beta \geq \operatorname{det} \omega>0 .
$$

Consequently, $\operatorname{det} R>0$ and $R$ is always non-singular when $\omega>0$.

Proof. (i) Set

$$
\sigma=\omega^{-\frac{1}{2}} \beta \omega^{-\frac{1}{2}} .
$$

Then $\sigma$ is skew-symmetric and

$$
R=\omega+\beta=\omega^{\frac{1}{2}}\left(E+\omega^{-\frac{1}{2}} \beta \omega^{-\frac{1}{2}}\right) \omega^{\frac{1}{2}}=\omega^{\frac{1}{2}}(E+\sigma) \omega^{\frac{1}{2}} .
$$

Set

$$
D_{1}=\operatorname{diag}\left(\mathrm{i} \sigma_{1}, \ldots, \mathrm{i} \sigma_{n}\right),
$$


where $\mathrm{i} \sigma_{1}, \ldots, \mathrm{i} \sigma_{n}$ are the eigenvalues of $\sigma, \mathrm{i}$ is the imaginary unit, $\sigma_{j} \in \mathbb{R}, j=$ $1, \ldots, n$ and

$$
\sigma_{2 j-1}=-\sigma_{2 j}, j=1,2, \ldots,\left[\frac{n}{2}\right] \text { and } \sigma_{n}=0 \text { if } n \text { is odd. }
$$

Then we can write for some unitary matrix $C_{1} \in \mathbb{C}^{n \times n}$,

$$
\sigma=C_{1} D_{1} C_{1}^{*},
$$

where $C_{1}^{*}$ is the Hermitian adjoint of $C_{1}, C_{1}^{*}=C_{1}^{-1}$. It follows from (3.2) and (3.5) that

$$
R=\omega^{\frac{1}{2}} C_{1}\left(E+D_{1}\right) C_{1}^{*} \omega^{\frac{1}{2}},
$$

which together with (3.3) and (3.4) yields

$$
\operatorname{det} R=(\operatorname{det} \omega)\left(1+\sigma_{1}^{2}\right)\left(1+\sigma_{3}^{2}\right) \cdots\left(1+\sigma_{2\left[\frac{n}{2}\right]-1}^{2}\right) .
$$

Also from (3.4), we have

$$
\operatorname{det} \sigma=0 \text { if } n \text { is odd, } \operatorname{det} \sigma=\sigma_{1}^{2} \sigma_{3}^{2} \cdots \sigma_{n-1}^{2} \text { if } n \text { is even. }
$$

It follows that

$$
0 \leq \operatorname{det} \sigma \leq \sigma_{1}^{2} \sigma_{3}^{2} \cdots \sigma_{2\left[\frac{n}{2}\right]-1}^{2}
$$

This together with (3.1) gives

$$
\operatorname{det} \beta=(\operatorname{det} \omega)(\operatorname{det} \sigma) \geq 0 .
$$

Combining (3.6)-(3.8), we obtain

$$
\operatorname{det} R \geq(\operatorname{det} \omega)(1+\operatorname{det} \sigma)=\operatorname{det} \omega+\operatorname{det} \beta \geq \operatorname{det} \omega>0 .
$$

(ii) When $n=2, \operatorname{det} \sigma=\sigma_{1}^{2}$. We infer from this, (3.6) and (3.8) that $\operatorname{det} R=(\operatorname{det} \omega)\left(1+\sigma_{1}^{2}\right)=\operatorname{det} \omega+(\operatorname{det} \omega)(\operatorname{det} \sigma)=\operatorname{det} \omega+\operatorname{det} \beta \geq \operatorname{det} \omega>0$. The proof is completed.

Proposition 4. Suppose that $R=\omega+\beta \in D_{\delta, \mu}$ and the matrix $\sigma$ is given in (3.1). Then the following assertions hold:

(i) $\|\sigma\| \leq \delta<1$.

(ii) All eigenvalues $\mathrm{i} \sigma_{j}$ of $\sigma$ satisfy: $\left|\sigma_{j}\right| \leq \delta<1, j=1, \ldots, n$.

Proof. (i) Since $R=\omega+\beta \in D_{\delta, \mu}$, we have $\delta \lambda_{\min }(\omega) \geq \mu$ and $\|\beta\| \leq \mu$. From these estimates and (3.1), we obtain

$$
\|\sigma\| \leq\left\|\omega^{-\frac{1}{2}}\right\|^{2}\|\beta\| \leq \frac{1}{\lambda_{\min }(\omega)} \mu \leq \delta<1 .
$$

(ii) The estimate (ii) follows directly from (i) and the fact that $\left|\sigma_{j}\right| \leq\|\sigma\|, j=$ $1, \ldots, n$. 
Proposition 5. Suppose that $R=\omega+\beta \in D_{\delta, \mu}$. Then

$(3.9)$ $\frac{1}{\delta^{n}}\|\beta\|^{n}+\left(2^{\left[\frac{n}{2}\right]}-1\right) \operatorname{det} \beta \leq \operatorname{det} \omega+\left(2^{\left[\frac{n}{2}\right]}-1\right) \operatorname{det} \beta \leq \operatorname{det} R \leq\left(1+\delta^{2}\right)^{\left[\frac{n}{2}\right]} \operatorname{det} \omega$, where, when $\delta=0$ we have $\beta=0$ and $\frac{0}{0}=0$.

Proof. By (3.6),

$$
\operatorname{det} R=(\operatorname{det} \omega)\left(1+\sigma_{1}^{2}\right)\left(1+\sigma_{3}^{2}\right) \cdots\left(1+\sigma_{2\left[\frac{n}{2}\right]-1}^{2}\right) .
$$

By Proposition 4, $\left|\sigma_{j}\right| \leq \delta<1, j=1, \ldots, n$. Thus

$$
\begin{aligned}
\left(1+\delta^{2}\right)^{\left[\frac{n}{2}\right]} & \geq\left(1+\sigma_{1}^{2}\right)\left(1+\sigma_{3}^{2}\right) \cdots\left(1+\sigma_{2\left[\frac{n}{2}\right]-1}^{2}\right) \\
& \geq 1+\left(2^{\left[\frac{n}{2}\right]}-1\right) \sigma_{1}^{2} \sigma_{3}^{2} \cdots \sigma_{2\left[\frac{n}{2}\right]-1}^{2} \geq 1+\left(2^{\left[\frac{n}{2}\right]}-1\right) \operatorname{det} \sigma,
\end{aligned}
$$

where the last inequality is by (3.7). Moreover, we have

$$
\operatorname{det} \omega \geq\left(\lambda_{\min }(\omega)\right)^{n} \geq \frac{1}{\delta^{n}} \mu^{n} \geq \frac{1}{\delta^{n}}\|\beta\|^{n} .
$$

From these estimates and (3.8), we obtain the conclusion of Proposition 5 ,

Proposition 6. Suppose that $R=\omega+\beta \in D_{\delta, \mu}$ and the matrix $\sigma$ is given in (3.1). Then

$$
\begin{aligned}
& \frac{R^{-1}+\left(R^{-1}\right)^{T}}{2}=\omega^{-\frac{1}{2}}\left(E-\sigma^{2}\right)^{-1} \omega^{-\frac{1}{2}}, \\
& \frac{R^{-1}-\left(R^{-1}\right)^{T}}{2}=\omega^{-\frac{1}{2}}(-\sigma)\left(E-\sigma^{2}\right)^{-1} \omega^{-\frac{1}{2}} .
\end{aligned}
$$

Proof. It follows from (3.2) that

$$
\begin{gathered}
R^{-1}=\omega^{-\frac{1}{2}}(E+\sigma)^{-1} \omega^{-\frac{1}{2}} \\
\left(R^{-1}\right)^{T}=\omega^{-\frac{1}{2}}\left((E+\sigma)^{-1}\right)^{T} \omega^{-\frac{1}{2}}=\omega^{-\frac{1}{2}}(E-\sigma)^{-1} \omega^{-\frac{1}{2}} .
\end{gathered}
$$

Thus

$$
\begin{aligned}
& \frac{R^{-1}+\left(R^{-1}\right)^{T}}{2}=\omega^{-\frac{1}{2}} \frac{(E+\sigma)^{-1}+(E-\sigma)^{-1}}{2} \omega^{-\frac{1}{2}}, \\
& \frac{R^{-1}-\left(R^{-1}\right)^{T}}{2}=\omega^{-\frac{1}{2}} \frac{(E+\sigma)^{-1}-(E-\sigma)^{-1}}{2} \omega^{-\frac{1}{2}} .
\end{aligned}
$$

Note that $E-\sigma^{2}=(E-\sigma)(E+\sigma)$, so we have

$$
\begin{aligned}
& \frac{(E+\sigma)^{-1}+(E-\sigma)^{-1}}{2}\left(E-\sigma^{2}\right)=\frac{(E-\sigma)+(E+\sigma)}{2}=E, \\
& \frac{(E+\sigma)^{-1}-(E-\sigma)^{-1}}{2}\left(E-\sigma^{2}\right)=\frac{(E-\sigma)-(E+\sigma)}{2}=-\sigma .
\end{aligned}
$$


Therefore,

$$
\begin{aligned}
& \frac{(E+\sigma)^{-1}+(E-\sigma)^{-1}}{2}=\left(E-\sigma^{2}\right)^{-1}, \\
& \frac{(E+\sigma)^{-1}-(E-\sigma)^{-1}}{2}=(-\sigma)\left(E-\sigma^{2}\right)^{-1} .
\end{aligned}
$$

From these equalities and (3.11), we obtain the desired equalities in (3.10).

Corollary 1. Suppose that $R=\omega+\beta \in D_{\delta, \mu}$ and suppose that the matrix $\sigma=\omega^{-\frac{1}{2}} \beta \omega^{-\frac{1}{2}}$ is diagonalised by a unitary matrix $C_{1} \in \mathbb{C}^{n \times n}$ as in (3.5),

$$
\sigma=C_{1} D_{1} C_{1}^{*}
$$

where $D_{1}$ is the diagonal matrix given by (3.3).

Then

$$
\begin{aligned}
& \frac{R^{-1}+\left(R^{-1}\right)^{T}}{2}=\omega^{-\frac{1}{2}} C_{1} D_{2} C_{1}^{*} \omega^{-\frac{1}{2}} \\
& \frac{R^{-1}-\left(R^{-1}\right)^{T}}{2}=\omega^{-\frac{1}{2}} C_{1} D_{3} C_{1}^{*} \omega^{-\frac{1}{2}}
\end{aligned}
$$

where

$$
\begin{aligned}
& D_{2}=\left(E-D_{1}^{2}\right)^{-1}=\operatorname{diag}\left(\frac{1}{1+\sigma_{1}^{2}}, \ldots, \frac{1}{1+\sigma_{n}^{2}}\right), \\
& D_{3}=\left(-D_{1}\right)\left(E-D_{1}^{2}\right)^{-1}=\operatorname{diag}\left(\frac{-\mathrm{i} \sigma_{1}}{1+\sigma_{1}^{2}}, \ldots, \frac{-\mathrm{i} \sigma_{n}}{1+\sigma_{n}^{2}}\right) .
\end{aligned}
$$

Proof. All equalities in (3.12), (3.13) are followed easily from (3.3), (3.5) and (3.10).

Corollary 2. Suppose that $R=\omega+\beta \in D_{\delta, \mu}$. Then

$$
\frac{1}{1+\delta^{2}} \operatorname{Tr} \omega^{-1} \leq \operatorname{Tr} R^{-1} \leq \operatorname{Tr} \omega^{-1}
$$

here and in the below, Tr stands for the trace operator of square matrices.

Proof. From (3.12) and (3.13), we have

$$
\begin{aligned}
\operatorname{Tr} R^{-1} & =\operatorname{Tr}\left(\frac{R^{-1}+\left(R^{-1}\right)^{T}}{2}\right)=\operatorname{Tr}\left(\omega^{-\frac{1}{2}} C_{1} D_{2} C_{1}^{*} \omega^{-\frac{1}{2}}\right) \\
& =\operatorname{Tr}\left(D_{2} C_{1}^{*} \omega^{-1} C_{1}\right)=\sum_{j}\left(D_{2}\right)_{j j}\left(C_{1}^{*} \omega^{-1} C_{1}\right)_{j j} .
\end{aligned}
$$

Note that $\omega^{-1}$ is positive definite, $C_{1}$ is unitary and, by Proposition $4, \frac{1}{1+\delta^{2}} \leq$ $\left(D_{2}\right)_{j j} \leq 1, j=1, \ldots, n$. We then obtain from (3.15) that

$$
\frac{1}{1+\delta^{2}} \operatorname{Tr} \omega^{-1} \leq \frac{1}{1+\delta^{2}} \sum_{j}\left(C_{1}^{*} \omega^{-1} C_{1}\right)_{j j} \leq \operatorname{Tr} R^{-1} \leq \sum_{j}\left(C_{1}^{*} \omega^{-1} C_{1}\right)_{j j}=\operatorname{Tr} \omega^{-1}
$$


This completes the proof.

3.2. The second differentials of the nonsymmetric Monge-Ampère type functions.

Proposition 7. Let $F(R)$ be the function given by (1.4), where $\operatorname{det} R>0$. Let $R^{-1}=\left[R^{i j}\right]$ denote the inverse of $R=\left[R_{i j}\right]$. Then for all $i, j, k, \ell=1, \ldots, n$, we have that

$$
\begin{aligned}
F^{i j} & \equiv \frac{\partial F(R)}{\partial R_{i j}}=R^{j i}, \\
F^{i j, k \ell} & \equiv \frac{\partial^{2} F(R)}{\partial R_{i j} \partial R_{k \ell}}=-R^{\ell i} R^{j k} .
\end{aligned}
$$

Proof. Let $U=\left[U_{i j}\right]$ denote the cofactor matrix of $R$, i.e., $U^{T}=(\operatorname{det} R) R^{-1}$. For a fixed $i, i=1, \ldots, n$, we expand the determinant $\operatorname{det} R$ according to the $i-$ th row,

$$
\operatorname{det} R=R_{i 1} U_{i 1}+\cdots+R_{i n} U_{i n}
$$

Then

$$
\frac{\partial F(R)}{\partial R_{i j}}=\frac{1}{\operatorname{det} R} \frac{\partial(\operatorname{det} R)}{\partial R_{i j}}=\frac{1}{\operatorname{det} R} U_{i j}=R^{j i}, \text { for } i, j=1, \ldots, n .
$$

Thus (3.16) is proved.

It follows from (3.16) that

$$
\sum_{p} R_{s p} F^{i p}=\sum_{p} R_{s p} R^{p i}=\delta_{i s}, \text { for } i, s=1, \ldots, n .
$$

Differentiating this equation with respect to $R_{k \ell}$, we get

$$
\sum_{p} \frac{\partial R_{s p}}{\partial R_{k \ell}} F^{i p}+\sum_{p} R_{s p} F^{i p, k \ell}=0
$$

and thus

$$
\delta_{s k} F^{i \ell}+\sum_{p} R_{s p} F^{i p, k \ell}=0, \text { for } i, s, k, \ell=1, \ldots, n .
$$

Multiplying this equality by $R^{j s}$ and summing over $s$, we have for $i, j, k, \ell=$ $1, \ldots, n$,

$$
\sum_{s} \delta_{s k} F^{i \ell} R^{j s}+\sum_{p, s} R^{j s} R_{s p} F^{i p, k \ell}=0
$$

or

$$
R^{\ell i} R^{j k}+F^{i j, k \ell}=0
$$

which gives the required result (3.17). The proof is now completed. 
Now we consider the second order differentials of the function $F(R)$ given by (1.4), where $R \in D_{\delta, \mu}, D_{\delta, \mu}$ is the unbounded and convex set given in (1.5). Let $M=\left[M_{i j}\right] \in \mathbb{R}^{n \times n}$. We consider the function $\mathcal{F}$ defined as follows:

$$
\begin{aligned}
\mathcal{F}(R, M) & : D_{\delta, \mu} \times \mathbb{R}^{n \times n} \rightarrow \mathbb{R}, \\
\mathcal{F}(R, M) & =\sum_{i, j, k, \ell} \frac{\partial^{2} F}{\partial R_{i j} \partial R_{k \ell}} M_{i j} M_{k \ell} \\
& =-\sum_{i, j, k, \ell} R^{\ell i} R^{j k} M_{i j} M_{k \ell} .
\end{aligned}
$$

Proposition 8. Suppose $R \in D_{\delta, \mu}$. Then for any matrix $M=P+Q \in \mathbb{R}^{n \times n}$, the following equality holds

$$
\mathcal{F}(R, M)=\mathcal{F}(R, P)+\mathcal{F}(R, Q)+2 \mathcal{L}(R, P, Q),
$$

where

$$
\mathcal{L}(R, P, Q)=-\sum_{i, j, k, \ell} R^{\ell i} R^{j k} P_{i j} Q_{k \ell} .
$$

Proof. It follows from (3.18) that

$$
\begin{aligned}
\mathcal{F}(R, M) & =-\sum_{i, j, k, \ell} R^{\ell i} R^{j k} P_{i j} P_{k \ell}-\sum_{i, j, k, \ell} R^{\ell i} R^{j k} Q_{i j} Q_{k \ell} \\
& -\sum_{i, j, k, \ell} R^{\ell i} R^{j k} P_{i j} Q_{k \ell}-\sum_{i, j, k, \ell} R^{\ell i} R^{j k} P_{k \ell} Q_{i j} .
\end{aligned}
$$

Note that

$$
\sum_{i, j, k, \ell} R^{\ell i} R^{j k} P_{k \ell} Q_{i j} \stackrel{\substack{\ell \leftrightarrow j \\ i \stackrel{\leftrightarrow}{=}}}{\sum_{i, j, k, \ell}} R^{j k} R^{\ell i} P_{i j} Q_{k \ell} .
$$

Combining these equalities, we obtain the conclusion of Proposition 8 ,

Proposition 9. Suppose that $R \in D_{\delta, \mu}$. Then for any symmetric matrix $P \in$ $\mathbb{R}^{n \times n}$, the following equality holds

$$
\mathcal{F}(R, P)=-[\mathcal{G}(R, P)]^{2}+\mathcal{H}(R, P),
$$

where

$$
\begin{aligned}
\mathcal{G}(R, P) & =\operatorname{Tr}\left(R^{-1} P\right), \\
\mathcal{H}(R, P) & =2 \operatorname{Tr}\left[\left(R^{-1}\right)^{(2)} P^{(2)}\right] .
\end{aligned}
$$

Proof. From (3.18) and the fact that $P^{T}=P$, we have

$$
\begin{aligned}
\mathcal{F}(R, P) & =-\sum_{i, j, k, \ell} R^{\ell i} R^{j k} P_{i j} P_{k \ell}=-\sum_{i, j, k, \ell} R^{\ell i} R^{j k} P_{j i} P_{\ell k} \\
& \stackrel{i \leftrightarrow \ell}{=}-\sum_{i, j, k, \ell} R^{i \ell} R^{k j} P_{k \ell} P_{i j} \stackrel{j \leftrightarrow \ell}{=}-\sum_{i, j, k, \ell} R^{i j} R^{k \ell} P_{k j} P_{i \ell} .
\end{aligned}
$$


It follows that

$$
\begin{aligned}
& \mathcal{F}(R, P)=-\frac{1}{2}\left(\sum_{i, j} R^{i j} P_{i j}\right)\left(\sum_{k, \ell} R^{k \ell} P_{k \ell}\right)-\frac{1}{2}\left(\sum_{i, \ell} R^{i \ell} P_{i \ell}\right)\left(\sum_{j, k} R^{k j} P_{k j}\right) \\
&+ \frac{1}{2}\left[\left(\sum_{i, j} R^{i j} P_{i j}\right)\left(\sum_{k, \ell} R^{k \ell} P_{k \ell}\right)+\left(\sum_{i, \ell} R^{i \ell} P_{i \ell}\right)\left(\sum_{j, k} R^{k j} P_{k j}\right)\right. \\
&\left.-\sum_{i, j, k, \ell} R^{i \ell} R^{k j} P_{k \ell} P_{i j}-\sum_{i, j, k, \ell} R^{i j} R^{k \ell} P_{k j} P_{i \ell}\right] \\
&=-\left(\sum_{i, j} R^{i j} P_{i j}\right)^{2}+2 \sum_{i<k}\left(R^{i j} R^{k \ell}-R^{i \ell} R^{k j}\right)\left(P_{i j} P_{k \ell}-P_{i \ell} P_{k j}\right) \\
&=-\left(\sum_{i, j} R^{i j} P_{i j}\right)^{2}+2 \sum_{\substack{i<k \\
j<\ell}}\left(\left(R^{-1}\right)_{i k, j \ell}^{(2)} P_{j \ell, i k}^{(2)}\right) \\
&=-\left[\operatorname{Tr}\left(R^{-1} P\right)\right]^{2}+2 \operatorname{Tr}\left[\left(R^{-1}\right)^{(2)} P^{(2)}\right] .
\end{aligned}
$$

This completes the proof.

Proposition 10. Suppose that $R \in D_{\delta, \mu}$. Then for any skew-symmetric matrix $Q \in \mathbb{R}^{n \times n}$, the following equality holds

$$
\mathcal{F}(R, Q)=-[\mathcal{G}(R, Q)]^{2}+\mathcal{H}(R, Q),
$$

where the functions $\mathcal{G}$ and $\mathcal{H}$ are defined as in (3.22).

Proof. The proof is similar to that of Proposition 9.

Proposition 11. Suppose that $R \in D_{\delta, \mu}$. Then for any symmetric matrix $P \in$ $\mathbb{R}^{n \times n}$ and any skew-symmetric matrix $Q \in \mathbb{R}^{n \times n}$, the following equality holds

$$
\mathcal{L}(R, P, Q)=-\frac{1}{2} \operatorname{Tr}\left[\left(R^{-1}-\left(R^{-1}\right)^{T}\right) P\left(R^{-1}+\left(R^{-1}\right)^{T}\right) Q\right],
$$

where $\mathcal{L}(R, P, Q)$ is defined by (3.20).

Proof. Note that

$$
\mathcal{L}(R, P, Q)=-\sum_{i, j, k, \ell} R^{\ell i} R^{j k} P_{i j} Q_{k \ell} \stackrel{i \leftrightarrow j}{k \stackrel{=}{k}}-\sum_{i, j, k, \ell} R^{k j} R^{i \ell} P_{j i} Q_{\ell k} .
$$


From this and the fact that $P^{T}=P, Q^{T}=-Q$, we get

$$
\begin{aligned}
& \mathcal{L}(R, P, Q)=-\frac{1}{2} \sum_{i, j, k, \ell} R^{\ell i} R^{j k} P_{i j} Q_{k \ell}-\frac{1}{2} \sum_{i, j, k, \ell} R^{k j} R^{i \ell} P_{j i} Q_{\ell k} \\
& =\frac{1}{2} \sum_{i, j, k, \ell} R^{j k} P_{j i} R^{\ell i} Q_{\ell k}-\frac{1}{2} \sum_{i, j, k, \ell} R^{k j} P_{j i} R^{i \ell} Q_{\ell k} \\
& =\frac{1}{2} \operatorname{Tr}\left[\left(R^{-1}\right)^{T} P\left(R^{-1}\right)^{T} Q\right]-\frac{1}{2} \operatorname{Tr}\left[R^{-1} P R^{-1} Q\right] \\
& =-\frac{1}{2} \operatorname{Tr}\left[\left(R^{-1}-\left(R^{-1}\right)^{T}\right) P\left(R^{-1}\right)^{T} Q\right]-\frac{1}{2} \operatorname{Tr}\left[\left(R^{-1}-\left(R^{-1}\right)^{T}\right) P R^{-1} Q\right] \\
& =-\frac{1}{2} \operatorname{Tr}\left[\left(R^{-1}-\left(R^{-1}\right)^{T}\right) P\left(\left(R^{-1}\right)^{T}+R^{-1}\right) Q\right],
\end{aligned}
$$

where in the fourth step, we have used the equality

$$
\operatorname{Tr}\left[R^{-1} P\left(R^{-1}\right)^{T} Q\right]=\operatorname{Tr}\left[\left(R^{-1}\right)^{T} P R^{-1} Q\right]=0,
$$

which holds due to the skew-symmetry of $Q$ and the symmetry of matrices $R^{-1} P\left(R^{-1}\right)^{T},\left(R^{-1}\right)^{T} P R^{-1}$. The proof is completed.

For $R=\omega+\beta \in D_{\delta, \mu}$ fixed and for matrix $M \in \mathbb{R}^{n \times n}$, we set

$$
\tilde{M} \equiv \omega^{-\frac{1}{2}} M \omega^{-\frac{1}{2}}=\left[\tilde{M}_{j k}\right], \quad \tilde{\tilde{M}} \equiv C_{1}^{*} \tilde{M} C_{1}=\left[\tilde{\tilde{M}}_{j k}\right],
$$

where $C_{1}$ is the unitary matrix defined in (3.5). It is obvious that

$$
|\tilde{M}|=|\tilde{\tilde{M}}|, \quad\|\tilde{M}\|=\|\tilde{\tilde{M}}\|,
$$

where $|\cdot|$ and $\|\cdot\|$ denote, respectively, the Frobenius norm and the operator norm on $\mathbb{C}^{n \times n}$, which are defined as follows: for any matrix $K=\left[K_{i j}\right] \in \mathbb{C}^{n \times n}$,

$$
|K|=\left(\sum_{i, j}\left|K_{i j}\right|^{2}\right)^{1 / 2}, \quad\|K\|=\sup _{\xi \in \mathbb{C}^{n},|\xi|=1}|K \xi| .
$$

Proposition 12. For any matrix $M \in \mathbb{R}^{n \times n}$, we have the following estimate

$$
\left(\lambda_{\max }(\omega)\right)^{-2}|M|^{2} \leq|\tilde{M}|^{2} \leq\left(\lambda_{\min }(\omega)\right)^{-2}|M|^{2},
$$

where $\lambda_{\max }(\omega)$ and $\lambda_{\min }(\omega)$ denote, respectively, the largest and smallest eigenvalues of $\omega$.

Proof. Let $\lambda_{1}, \ldots, \lambda_{n}$ be the eigenvalues of $\omega$, where $\lambda_{1} \geq \cdots \geq \lambda_{n}>0$. Write

$$
\omega=C D C^{-1},
$$

where $C$ is orthogonal and $D=\operatorname{diag}\left(\lambda_{1}, \ldots, \lambda_{n}\right)$. Then

$$
\omega^{-\frac{1}{2}}=C D^{-\frac{1}{2}} C^{-1}, D^{-\frac{1}{2}}=\operatorname{diag}\left(\lambda_{1}^{-\frac{1}{2}}, \ldots, \lambda_{n}^{-\frac{1}{2}}\right) .
$$


Therefore,

$$
\begin{aligned}
|\tilde{M}|^{2} & =\left|\omega^{-\frac{1}{2}} M \omega^{-\frac{1}{2}}\right|^{2}=\left|\left(C D^{-\frac{1}{2}} C^{-1}\right) M\left(C D^{-\frac{1}{2}} C^{-1}\right)\right|^{2} \\
& =\left|D^{-\frac{1}{2}}\left(C^{-1} M C\right) D^{-\frac{1}{2}}\right|^{2}=\sum_{i, j} \lambda_{i}^{-1} \lambda_{j}^{-1}\left(\left(C^{-1} M C\right)_{i j}\right)^{2} .
\end{aligned}
$$

From this and the fact that $0<\lambda_{1}^{-1} \leq \lambda_{i}^{-1} \leq \lambda_{n}^{-1}(i=1, \ldots, n)$, we obtain

$$
\lambda_{1}^{-2}|M|^{2}=\lambda_{1}^{-2} \sum_{i, j}\left(\left(C^{-1} M C\right)_{i j}\right)^{2} \leq|\tilde{M}|^{2} \leq \lambda_{n}^{-2} \sum_{i, j}\left(\left(C^{-1} M C\right)_{i j}\right)^{2}=\lambda_{n}^{-2}|M|^{2} .
$$

The proof is completed.

Proposition 13. Suppose that $R=\omega+\beta \in D_{\delta, \mu}$. Then for any symmetric matrix $P \in \mathbb{R}^{n \times n}$, we have

$$
\mathcal{F}(R, P)=-\sum_{j, k=1}^{n} \frac{1-\sigma_{j} \sigma_{k}}{\left(1+\sigma_{j}^{2}\right)\left(1+\sigma_{k}^{2}\right)}\left|\tilde{\tilde{P}}_{j k}\right|^{2}
$$

where $\mathrm{i} \sigma_{1}, \ldots, \mathrm{i} \sigma_{n}$ are the eigenvalues of the matrix $\sigma$, defined by (3.1).

Proof. Since $P$ is symmetric, by Proposition 1, $P^{(2)}$ is also symmetric. Hence from (2.1), (3.21) and (3.22), we have

$$
\begin{aligned}
& \mathcal{F}(R, P)=-[\mathcal{G}(R, P)]^{2}+\mathcal{H}(R, P)=-[\mathcal{G}(R, P)]^{2}+2 \operatorname{Tr}\left[\left(R^{-1}\right)^{(2)} P^{(2)}\right] \\
& =-[\mathcal{G}(R, P)]^{2}+2 \operatorname{Tr}\left[\frac{\left(R^{-1}\right)^{(2)}+\left(\left(R^{-1}\right)^{T}\right)^{(2)}}{2} P^{(2)}\right]=-[\mathcal{G}(R, P)]^{2} \\
& +2 \operatorname{Tr}\left[\left(\frac{R^{-1}+\left(R^{-1}\right)^{T}}{2}\right)^{(2)} P^{(2)}\right]+2 \operatorname{Tr}\left[\left(\frac{R^{-1}-\left(R^{-1}\right)^{T}}{2}\right)^{(2)} P^{(2)}\right] .
\end{aligned}
$$

It follows from (3.12) and (3.13) that

$$
\begin{aligned}
\mathcal{G}(R, P) & =\operatorname{Tr}\left(\frac{R^{-1}+\left(R^{-1}\right)^{T}}{2} P\right)=\operatorname{Tr}\left(\omega^{-\frac{1}{2}} C_{1} D_{2} C_{1}^{*} \omega^{-\frac{1}{2}} P\right) \\
& =\operatorname{Tr}\left(D_{2} C_{1}^{*} \omega^{-\frac{1}{2}} P \omega^{-\frac{1}{2}} C_{1}\right)=\operatorname{Tr}\left(D_{2} \tilde{\tilde{P}}\right)=\sum_{j} \frac{\tilde{\tilde{P}}_{j j}}{1+\sigma_{j}^{2}} .
\end{aligned}
$$


Since $P$ is symmetric, $\tilde{\tilde{P}}$ is Hermitian. Hence $\tilde{\tilde{P}}_{j k} \tilde{\tilde{P}}_{k j}=\left|\tilde{\tilde{P}}_{j k}\right|^{2}, j, k=1, \ldots, n$. From these equalities, (3.12), (3.13) and Proposition 1, we obtain

$$
\begin{aligned}
& 2 \operatorname{Tr}\left[\left(\frac{R^{-1}+\left(R^{-1}\right)^{T}}{2}\right)^{(2)} P^{(2)}\right]=2 \operatorname{Tr}\left[\left(\omega^{-\frac{1}{2}} C_{1} D_{2} C_{1}^{*} \omega^{-\frac{1}{2}}\right)^{(2)} P^{(2)}\right] \\
& =2 \operatorname{Tr}\left[D_{2}^{(2)}\left(C_{1}^{*}\right)^{(2)}\left(\omega^{-\frac{1}{2}}\right)^{(2)} P^{(2)}\left(\omega^{-\frac{1}{2}}\right)^{(2)} C_{1}^{(2)}\right] \\
& =2 \operatorname{Tr}\left[D_{2}^{(2)} \tilde{\tilde{P}}^{(2)}\right]=2 \sum_{j<k}\left(D_{2}^{(2)}\right)_{j k, j k} \tilde{\tilde{P}}_{j k, j k}^{(2)} \\
& =2 \sum_{j<k} \frac{\tilde{\tilde{P}}_{j j} \tilde{\tilde{P}}_{k k}-\left|\tilde{\tilde{P}}_{j k}\right|^{2}}{\left(1+\sigma_{j}^{2}\right)\left(1+\sigma_{k}^{2}\right)}=\sum_{j \neq k} \frac{\tilde{\tilde{P}}_{j j} \tilde{\tilde{P}}_{k k}}{\left(1+\sigma_{j}^{2}\right)\left(1+\sigma_{k}^{2}\right)}-\sum_{j \neq k} \frac{\left|\tilde{\tilde{P}}_{j k}\right|^{2}}{\left(1+\sigma_{j}^{2}\right)\left(1+\sigma_{k}^{2}\right)} \\
& =\sum_{j, k} \frac{\left|\tilde{\tilde{P}}_{j k}\right|^{2}}{\left(1+\sigma_{j}^{2}\right)\left(1+\sigma_{k}^{2}\right)}-\sum_{j, k} \frac{\mid\left(1+\sigma_{j}^{2}\right)\left(1+\sigma_{k}^{2}\right)}{\left(\left.\tilde{\tilde{P}}_{j k}\right|^{2}\right.} \\
& =\left(\sum_{j} \frac{\tilde{\tilde{P}}_{j j}}{1+\sigma_{j}^{2}}\right)^{2}-\sum_{j, k} \frac{\left(1+\sigma_{j}^{2}\right)\left(1+\sigma_{k}^{2}\right)}{(1+}
\end{aligned}
$$

Combining (3.30), (3.31) yields

$$
2 \operatorname{Tr}\left[\left(\frac{R^{-1}+\left(R^{-1}\right)^{T}}{2}\right)^{(2)} P^{(2)}\right]=[\mathcal{G}(R, P)]^{2}-\sum_{j, k} \frac{\left|\tilde{\tilde{P}}_{j k}\right|^{2}}{\left(1+\sigma_{j}^{2}\right)\left(1+\sigma_{k}^{2}\right)}
$$

From (3.12), (3.13) and Proposition 1, we also get

$$
\begin{aligned}
& 2 \operatorname{Tr}\left[\left(\frac{R^{-1}-\left(R^{-1}\right)^{T}}{2}\right)^{(2)} P^{(2)}\right]=2 \operatorname{Tr}\left[\left(\omega^{-\frac{1}{2}} C_{1} D_{3} C_{1}^{*} \omega^{-\frac{1}{2}}\right)^{(2)} P^{(2)}\right] \\
& =2 \operatorname{Tr}\left[D_{3}^{(2)} \tilde{\tilde{P}}^{(2)}\right]=2 \sum_{j<k}\left(D_{3}^{(2)}\right)_{j k, j k} \tilde{\tilde{P}}_{j k, j k}^{(2)} \\
& =2 \sum_{j<k} \frac{-\sigma_{j} \sigma_{k}}{\left(1+\sigma_{j}^{2}\right)\left(1+\sigma_{k}^{2}\right)}\left(\tilde{\tilde{P}}_{j j} \tilde{\tilde{P}}_{k k}-\left|\tilde{\tilde{P}}_{j k}\right|^{2}\right) \\
& =-\sum_{j \neq k} \frac{\sigma_{j} \sigma_{k}}{\left(1+\sigma_{j}^{2}\right)\left(1+\sigma_{k}^{2}\right)} \tilde{\tilde{P}}_{j j} \tilde{\tilde{P}}_{k k}+\sum_{j \neq k} \frac{\sigma_{j} \sigma_{k}}{\left(1+\sigma_{j}^{2}\right)\left(1+\sigma_{k}^{2}\right)}\left|\tilde{\tilde{P}}_{j k}\right|^{2} .
\end{aligned}
$$


Obviously, $\operatorname{Tr}\left(\frac{R^{-1}-\left(R^{-1}\right)^{T}}{2} P\right)=0$. It then follows from (3.12) and (3.13) that

$$
\operatorname{Tr}\left(\omega^{-\frac{1}{2}} C_{1} D_{3} C_{1}^{*} \omega^{-\frac{1}{2}} P\right)=\operatorname{Tr}\left(D_{3} \tilde{\tilde{P}}\right)=\sum_{j} \frac{-\mathrm{i} \sigma_{j}}{1+\sigma_{j}^{2}} \tilde{\tilde{P}}_{j j}=0
$$

or equivalently,

$$
\left(\sum_{j} \frac{\sigma_{j}}{1+\sigma_{j}^{2}} \tilde{\tilde{P}}_{j j}\right)^{2}=0
$$

Hence

$$
\sum_{j} \frac{\sigma_{j}^{2}}{\left(1+\sigma_{j}^{2}\right)^{2}}\left(\tilde{\tilde{P}}_{j j}\right)^{2}=-\sum_{j \neq k} \frac{\sigma_{j} \sigma_{k}}{\left(1+\sigma_{j}^{2}\right)\left(1+\sigma_{k}^{2}\right)} \tilde{\tilde{P}}_{j j} \tilde{\tilde{P}}_{k k}
$$

This together with (3.33) gives

$$
2 \operatorname{Tr}\left[\left(\frac{R^{-1}-\left(R^{-1}\right)^{T}}{2}\right)^{(2)} P^{(2)}\right]=\sum_{j, k} \frac{\sigma_{j} \sigma_{k}}{\left(1+\sigma_{j}^{2}\right)\left(1+\sigma_{k}^{2}\right)}\left|\tilde{\tilde{P}}_{j k}\right|^{2} .
$$

The proof is straightforward from (3.29), (3.32) and (3.34).

Corollary 3. Suppose that $R=\omega+\beta \in D_{\delta, \mu}$. Then for any symmetric matrix $P \in \mathbb{R}^{n \times n}$, we have

$$
\mathcal{F}(R, P) \leq-\frac{1-\delta^{2}}{\left(1+\delta^{2}\right)^{2}}|\tilde{P}|^{2} \leq-\frac{1-\delta^{2}}{\left(1+\delta^{2}\right)^{2}}\left(\lambda_{\max }(\omega)\right)^{-2}|P|^{2} .
$$

Proof. By Proposition 4, we have $\left|\sigma_{j}\right| \leq \delta<1, j=1, \ldots, n$. Hence from (3.26) and (3.28), we obtain

$$
\begin{aligned}
\mathcal{F}(R, P) & =-\sum_{j, k} \frac{1-\sigma_{j} \sigma_{k}}{\left(1+\sigma_{j}^{2}\right)\left(1+\sigma_{k}^{2}\right)}\left|\tilde{\tilde{P}}_{j k}\right|^{2} \leq-\sum_{j, k} \frac{1-\left|\sigma_{j}\right|\left|\sigma_{k}\right|}{\left(1+\sigma_{j}^{2}\right)\left(1+\sigma_{k}^{2}\right)}\left|\tilde{\tilde{P}}_{j k}\right|^{2} \\
& \leq-\frac{1-\delta^{2}}{\left(1+\delta^{2}\right)^{2}} \sum_{j, k}\left|\tilde{\tilde{P}}_{j k}\right|^{2}=-\frac{1-\delta^{2}}{\left(1+\delta^{2}\right)^{2}}|\tilde{\tilde{P}}|^{2}=-\frac{1-\delta^{2}}{\left(1+\delta^{2}\right)^{2}}|\tilde{P}|^{2}
\end{aligned}
$$

Thus we get the first inequality in (3.35). Combining this with Proposition 12 , we can easily obtain the second inequality in (3.35).

Proposition 14. Suppose that $R=\omega+\beta \in D_{\delta, \mu}$. Then for any skew-symmetric matrix $Q \in \mathbb{R}^{n \times n}$, we have

$$
\mathcal{F}(R, Q)=\sum_{j, k=1}^{n} \frac{1-\sigma_{j} \sigma_{k}}{\left(1+\sigma_{j}^{2}\right)\left(1+\sigma_{k}^{2}\right)}\left|\tilde{\tilde{Q}}_{j k}\right|^{2} .
$$


Proof. Since $Q$ is skew-symmetric, by Proposition $1, Q^{(2)}$ is symmetric. By arguing as in (3.29), we obtain from (3.23),

$$
\begin{aligned}
& \mathcal{F}(R, Q)=-[\mathcal{G}(R, Q)]^{2}+\mathcal{H}(R, Q)=-[\mathcal{G}(R, Q)]^{2} \\
& +2 \operatorname{Tr}\left[\left(\frac{R^{-1}+\left(R^{-1}\right)^{T}}{2}\right)^{(2)} Q^{(2)}\right]+2 \operatorname{Tr}\left[\left(\frac{R^{-1}-\left(R^{-1}\right)^{T}}{2}\right)^{(2)} Q^{(2)}\right] .
\end{aligned}
$$

It follows from (3.12) and (3.13) that

$$
\begin{aligned}
\mathcal{G}(R, Q) & =\operatorname{Tr}\left(\frac{R^{-1}-\left(R^{-1}\right)^{T}}{2} Q\right)=\operatorname{Tr}\left(\omega^{-\frac{1}{2}} C_{1} D_{3} C_{1}^{*} \omega^{-\frac{1}{2}} Q\right) \\
& =\operatorname{Tr}\left(D_{3} C_{1}^{*} \omega^{-\frac{1}{2}} Q \omega^{-\frac{1}{2}} C_{1}\right)=\operatorname{Tr}\left(D_{3} \tilde{\tilde{Q}}\right)=\sum_{j} \frac{-\mathrm{i} \sigma_{j}}{1+\sigma_{j}^{2}} \tilde{\tilde{Q}}_{j j}
\end{aligned}
$$

Since $Q$ is skew-symmetric, $\tilde{\tilde{Q}}$ is skew-Hermitian. Hence $\tilde{\tilde{Q}}_{j k} \tilde{\tilde{Q}}_{k j}=-\left|\tilde{\tilde{Q}}_{j k}\right|^{2}$, $j, k=1, \ldots, n$. From these equalities, (3.12), (3.13) and Proposition 1, we obtain

$$
\begin{aligned}
2 \operatorname{Tr} & {\left[\left(\frac{R^{-1}-\left(R^{-1}\right)^{T}}{2}\right)^{(2)} Q^{(2)}\right]=2 \operatorname{Tr}\left[\left(\omega^{-\frac{1}{2}} C_{1} D_{3} C_{1}^{*} \omega^{-\frac{1}{2}}\right)^{(2)} Q^{(2)}\right] } \\
& =2 \operatorname{Tr}\left[D_{3}^{(2)} \tilde{\tilde{Q}}^{(2)}\right]=2 \sum_{j<k}\left(D_{3}^{(2)}\right)_{j k, j k} \tilde{\tilde{Q}}_{j k, j k}^{(2)} \\
& =2 \sum_{j<k} \frac{-\sigma_{j} \sigma_{k}}{\left(1+\sigma_{j}^{2}\right)\left(1+\sigma_{k}^{2}\right)}\left(\tilde{\tilde{Q}}_{j j} \tilde{\tilde{Q}}_{k k}+\left|\tilde{\tilde{Q}}_{j k}\right|^{2}\right) \\
& =\sum_{j \neq k} \frac{-\sigma_{j} \sigma_{k}}{\left(1+\sigma_{j}^{2}\right)\left(1+\sigma_{k}^{2}\right)} \tilde{\tilde{Q}}_{j j} \tilde{\tilde{Q}}_{k k}+\sum_{j \neq k} \frac{-\sigma_{j} \sigma_{k}}{\left(1+\sigma_{j}^{2}\right)\left(1+\sigma_{k}^{2}\right)}\left|\tilde{\tilde{Q}}_{j k}\right|^{2} \\
& =\sum_{j, k} \frac{-\sigma_{j} \sigma_{k}}{\left(1+\sigma_{j}^{2}\right)\left(1+\sigma_{k}^{2}\right)} \tilde{\tilde{Q}}_{j j} \tilde{\tilde{Q}}_{k k}+\sum_{j, k} \frac{-\sigma_{j} \sigma_{k}}{\left(1+\sigma_{j}^{2}\right)\left(1+\sigma_{k}^{2}\right)}\left|\tilde{Q}_{j k}\right|^{2} .
\end{aligned}
$$

Combining (3.38), (3.39) gives

$$
2 \operatorname{Tr}\left[\left(\frac{R^{-1}-\left(R^{-1}\right)^{T}}{2}\right)^{(2)} Q^{(2)}\right]=[\mathcal{G}(R, Q)]^{2}+\sum_{j, k} \frac{-\sigma_{j} \sigma_{k}}{\left(1+\sigma_{j}^{2}\right)\left(1+\sigma_{k}^{2}\right)}\left|\tilde{Q}_{j k}\right|^{2} .
$$


From the equalities $\tilde{\tilde{Q}}_{j k} \tilde{\tilde{Q}}_{k j}=-\left|\tilde{\tilde{Q}}_{j k}\right|^{2}(j, k=1, \ldots, n),(\underline{3.12})$, (3.13) and Proposition 1, we also get

$$
\begin{aligned}
& 2 \operatorname{Tr}\left[\left(\frac{R^{-1}+\left(R^{-1}\right)^{T}}{2}\right)^{(2)} Q^{(2)}\right]=2 \operatorname{Tr}\left[\left(\omega^{-\frac{1}{2}} C_{1} D_{2} C_{1}^{*} \omega^{-\frac{1}{2}}\right)^{(2)} Q^{(2)}\right] \\
& =2 \operatorname{Tr}\left[D_{2}^{(2)} \tilde{\tilde{Q}}^{(2)}\right]=2 \sum_{j<k} \frac{1}{\left(1+\sigma_{j}^{2}\right)\left(1+\sigma_{k}^{2}\right)}\left(\tilde{\tilde{Q}}_{j j} \tilde{\tilde{Q}}_{k k}+\left|\tilde{\tilde{Q}}_{j k}\right|^{2}\right) \\
& =\sum_{j \neq k} \frac{1}{\left(1+\sigma_{j}^{2}\right)\left(1+\sigma_{k}^{2}\right)} \tilde{\tilde{Q}}_{j j} \tilde{\tilde{Q}}_{k k}+\sum_{j \neq k} \frac{1}{\left(1+\sigma_{j}^{2}\right)\left(1+\sigma_{k}^{2}\right)}\left|\tilde{\tilde{Q}}_{j k}\right|^{2} \\
& =\sum_{j, k} \frac{1}{\left(1+\sigma_{j}^{2}\right)\left(1+\sigma_{k}^{2}\right)} \tilde{\tilde{Q}}_{j j} \tilde{\tilde{Q}}_{k k}+\sum_{j, k} \frac{1}{\left(1+\sigma_{j}^{2}\right)\left(1+\sigma_{k}^{2}\right)}\left|\tilde{\tilde{Q}}_{j k}\right|^{2} \\
& =\left(\sum_{j} \frac{\tilde{\tilde{Q}}_{j j}}{1+\sigma_{j}^{2}}\right)^{2}+\sum_{j, k} \frac{1}{\left(1+\sigma_{j}^{2}\right)\left(1+\sigma_{k}^{2}\right)}\left|\tilde{\tilde{Q}}_{j k}\right|^{2} \text {. }
\end{aligned}
$$

Obviously, $\operatorname{Tr}\left(\frac{R^{-1}+\left(R^{-1}\right)^{T}}{2} Q\right)=0$. It follows from this, (3.12) and (3.13) that

$$
\operatorname{Tr}\left(\omega^{-\frac{1}{2}} C_{1} D_{2} C_{1}^{*} \omega^{-\frac{1}{2}} Q\right)=\operatorname{Tr}\left(D_{2} \tilde{\tilde{Q}}\right)=\sum_{j} \frac{\tilde{\tilde{Q}}_{j j}}{1+\sigma_{j}^{2}}=0 .
$$

Combining this and (3.41) gives

$$
2 \operatorname{Tr}\left[\left(\frac{R^{-1}+\left(R^{-1}\right)^{T}}{2}\right)^{(2)} Q^{(2)}\right]=\sum_{j, k} \frac{1}{\left(1+\sigma_{j}^{2}\right)\left(1+\sigma_{k}^{2}\right)}\left|\tilde{Q}_{j k}\right|^{2} .
$$

The proof is straightforward from (3.37), (3.40) and (3.42).

Corollary 4. Suppose that $R=\omega+\beta \in D_{\delta, \mu}$. Then for any skew-symmetric matrix $Q \in \mathbb{R}^{n \times n}$, we have

$$
\mathcal{F}(R, Q) \leq|\tilde{Q}|^{2}
$$

Proof. Note that

$$
\frac{1-\sigma_{j} \sigma_{k}}{\left(1+\sigma_{j}^{2}\right)\left(1+\sigma_{k}^{2}\right)} \leq \frac{1+\left|\sigma_{j}\right|\left|\sigma_{k}\right|}{\left(1+\sigma_{j}^{2}\right)\left(1+\sigma_{k}^{2}\right)} \leq 1, j, k=1, \ldots, n .
$$

From this, (3.26) and (3.36), we obtain that

$$
\mathcal{F}(R, Q) \leq \sum_{j, k}\left|\tilde{\tilde{Q}}_{j k}\right|^{2}=|\tilde{\tilde{Q}}|^{2}=|\tilde{Q}|^{2}
$$

This completes the proof. 
Proposition 15. Suppose that $R=\omega+\beta \in D_{\delta, \mu}$. Then for any symmetric matrix $P \in \mathbb{R}^{n \times n}$ and any skew-symmetric matrix $Q \in \mathbb{R}^{n \times n}$, we have

$$
|\mathcal{L}(R, P, Q)| \leq \frac{2 n \delta}{1+\delta^{2}}|\tilde{P}||\tilde{Q}|
$$

Proof. By (3.10) and (3.24), we have

$$
\begin{aligned}
\mathcal{L}(R, P, Q) & =-2 \operatorname{Tr}\left[\frac{\left(R^{-1}\right)-\left(R^{-1}\right)^{T}}{2} P \frac{\left(R^{-1}\right)+\left(R^{-1}\right)^{T}}{2} Q\right] \\
& =-2 \operatorname{Tr}\left[\left(\omega^{-\frac{1}{2}}(-\sigma)\left(E-\sigma^{2}\right)^{-1} \omega^{-\frac{1}{2}}\right) P\left(\omega^{-\frac{1}{2}}\left(E-\sigma^{2}\right)^{-1} \omega^{-\frac{1}{2}}\right) Q\right] \\
& =2 \operatorname{Tr}\left[\sigma\left(E-\sigma^{2}\right)^{-1}\left(\omega^{-\frac{1}{2}} P \omega^{-\frac{1}{2}}\right)\left(E-\sigma^{2}\right)^{-1}\left(\omega^{-\frac{1}{2}} Q \omega^{-\frac{1}{2}}\right)\right] \\
= & 2 \operatorname{Tr}\left[\sigma\left(E-\sigma^{2}\right)^{-1} \tilde{P}\left(E-\sigma^{2}\right)^{-1} \tilde{Q}\right] .
\end{aligned}
$$

Hence

$$
|\mathcal{L}(R, P, Q)| \leq 2\left|\sigma\left(E-\sigma^{2}\right)^{-1}\right|\left|\left(E-\sigma^{2}\right)^{-1}\right||\tilde{P}||\tilde{Q}| .
$$

From (3.3), (3.5) and Proposition 4, we can easily obtain

$$
\begin{gathered}
\left|\sigma\left(E-\sigma^{2}\right)^{-1}\right|=\left|D_{1}\left(E-D_{1}^{2}\right)^{-1}\right|=\left(\sum_{j} \frac{\sigma_{j}^{2}}{\left(1+\sigma_{j}^{2}\right)^{2}}\right)^{1 / 2} \leq \frac{\sqrt{n} \delta}{1+\delta^{2}}, \\
\left|\left(E-\sigma^{2}\right)^{-1}\right|=\left|\left(E-D_{1}^{2}\right)^{-1}\right|=\left(\sum_{j} \frac{1}{\left(1+\sigma_{j}^{2}\right)^{2}}\right)^{1 / 2} \leq \sqrt{n}
\end{gathered}
$$

Combining these estimates with (3.45), we get the desired estimate (3.44).

In the next theorem we will give an upper estimate for second-order differentials of the function $F(R)$.

Theorem 1. Suppose that $R=\omega+\beta \in D_{\delta, \mu}$. Then for any matrix $M=P+Q$, where $P \in \mathbb{R}^{n \times n}$ is symmetric and $Q \in \mathbb{R}^{n \times n}$ is skew-symmetric, we have

$$
\mathcal{F}(R, M) \leq-(1-\eta) \frac{1-\delta^{2}}{\left(1+\delta^{2}\right)^{2}}|\tilde{P}|^{2}+\left(1+\frac{4 n^{2} \delta^{2}}{\eta\left(1-\delta^{2}\right)}\right)|\tilde{Q}|^{2},
$$

for any constant $\eta \in(0,1]$, where $\tilde{P}=\omega^{-\frac{1}{2}} P \omega^{-\frac{1}{2}}, \tilde{Q}=\omega^{-\frac{1}{2}} Q \omega^{-\frac{1}{2}}$.

Proof. From (3.19), (3.35), (3.43) and (3.44), we have

$$
\begin{aligned}
\mathcal{F}(R, M) & =\mathcal{F}(R, P)+\mathcal{F}(R, Q)+2 \mathcal{L}(R, P, Q) \\
& \leq-\frac{1-\delta^{2}}{\left(1+\delta^{2}\right)^{2}}|\tilde{P}|^{2}+|\tilde{Q}|^{2}+\frac{4 n \delta}{1+\delta^{2}}|\tilde{P}||\tilde{Q}| .
\end{aligned}
$$


By using Cauchy's inequality, we have for any positive constant $\eta \in(0,1]$,

$$
\frac{4 n \delta}{1+\delta^{2}}|\tilde{P}||\tilde{Q}| \leq \frac{\eta\left(1-\delta^{2}\right)}{\left(1+\delta^{2}\right)^{2}}|\tilde{P}|^{2}+\frac{4 n^{2} \delta^{2}}{\eta\left(1-\delta^{2}\right)}|\tilde{Q}|^{2} .
$$

Combining these estimates, we obtain the estimate (3.46). The proof is completed.

\subsection{The $d$-concavity of the function $F(R)$.}

Theorem 2. For any matrices $R^{(0)}=\omega^{(0)}+\beta^{(0)}=\left[R_{i j}^{(0)}\right], R^{(1)}=\omega^{(1)}+\beta^{(1)}=$ $\left[R_{i j}^{(1)}\right]$ from the set $D_{\delta, \mu}$, we have

$$
\begin{aligned}
F\left(R^{(1)}\right)-F\left(R^{(0)}\right) & \leq \sum_{i, j=1}^{n} \frac{\partial F\left(R^{(0)}\right)}{\partial R_{i j}}\left(R_{i j}^{(1)}-R_{i j}^{(0)}\right) \\
& +\frac{1}{2}\left(1+\frac{4 n^{2} \delta^{2}}{1-\delta^{2}}\right)\left(\lambda_{\min }\left(\omega^{(s)}\right)\right)^{-2}\left|\beta^{(1)}-\beta^{(0)}\right|^{2},
\end{aligned}
$$

where $\omega^{(s)} \equiv(1-s) \omega^{(0)}+s \omega^{(1)}$ for some constant $s \in(0,1)$.

Proof. We set for all $t \in[0,1]$,

$$
g(t):=F\left((1-t) R^{(0)}+t R^{(1)}\right)=F\left(R^{(t)}\right),
$$

where $R^{(t)} \equiv(1-t) R^{(0)}+t R^{(1)}=\omega^{(t)}+\beta^{(t)}, \omega^{(t)}=(1-t) \omega^{(0)}+t \omega^{(1)}, \beta^{(t)}=$ $(1-t) \beta^{(0)}+t \beta^{(1)}$. Since $D_{\delta, \mu}$ is convex, we infer that $R^{(t)} \in D_{\delta, \mu}$.

By the Taylor expansion, we have for some constant $s \in(0,1)$,

$$
F\left(R^{(1)}\right)-F\left(R^{(0)}\right)=g(1)-g(0)=g^{\prime}(0)+\frac{1}{2} g^{\prime \prime}(s) .
$$

By computation, we have for all $t \in(0,1)$,

$$
\begin{aligned}
g^{\prime}(t) & =\sum_{i, j} \frac{\partial F\left(R^{(t)}\right)}{\partial R_{i j}}\left(R_{i j}^{(1)}-R_{i j}^{(0)}\right), \\
g^{\prime \prime}(t) & =\sum_{i, j, k, \ell} \frac{\partial^{2} F\left(R^{(t)}\right)}{\partial R_{i j} \partial R_{k \ell}}\left(R_{i j}^{(1)}-R_{i j}^{(0)}\right)\left(R_{k \ell}^{(1)}-R_{k \ell}^{(0)}\right)=\mathcal{F}\left(R^{(t)}, R^{(1)}-R^{(0)}\right),
\end{aligned}
$$

where the function $\mathcal{F}$ is defined by (3.18). Hence

$$
g^{\prime}(0)=\sum_{i, j} \frac{\partial F\left(R^{(0)}\right)}{\partial R_{i j}}\left(R_{i j}^{(1)}-R_{i j}^{(0)}\right) .
$$


Moreover, by applying Theorem 1 with $R=R^{(s)}=\omega^{(s)}+\beta^{(s)}, M=R^{(1)}-R^{(0)}=$ $\left(\omega^{(1)}-\omega^{(0)}\right)+\left(\beta^{(1)}-\beta^{(0)}\right) \equiv P+Q$ and $\eta=1$, we obtain

$$
\begin{aligned}
g^{\prime \prime}(s)=\mathcal{F}\left(R^{(s)}, R^{(1)}-R^{(0)}\right) & \leq\left(1+\frac{4 n^{2} \delta^{2}}{1-\delta^{2}}\right)\left|\left(\omega^{(s)}\right)^{-\frac{1}{2}}\left(\beta^{(1)}-\beta^{(0)}\right)\left(\omega^{(s)}\right)^{-\frac{1}{2}}\right|^{2} \\
& \leq\left(1+\frac{4 n^{2} \delta^{2}}{1-\delta^{2}}\right)\left(\lambda_{\min }\left(\omega^{(s)}\right)\right)^{-2}\left|\beta^{(1)}-\beta^{(0)}\right|^{2},
\end{aligned}
$$

where the last inequality is by Proposition 12, Combining this estimate with (3.48) and (3.49), we arrive at the estimate (3.47).

Now, we obtain the following theorem on $d$-concavity in the set $D_{\delta, \mu}$ for the Monge-Ampère type function $F(R)$.

Theorem 3. The function $F(R)=\log (\operatorname{det} R)$ is $d$-concave in the set $D_{\delta, \mu}$, where $d=2 n \delta^{2}\left(1+\frac{4 n^{2} \delta^{2}}{1-\delta^{2}}\right)$, depending only on $\delta$ and $n$. That means, for any matrices $R^{(0)}=\omega^{(0)}+\beta^{(0)}=\left[R_{i j}^{(0)}\right], R^{(1)}=\omega^{(1)}+\beta^{(1)}=\left[R_{i j}^{(1)}\right]$ from $D_{\delta, \mu}$, we have

$$
F\left(R^{(1)}\right)-F\left(R^{(0)}\right) \leq \sum_{i, j=1}^{n} \frac{\partial F\left(R^{(0)}\right)}{\partial R_{i j}}\left(R_{i j}^{(1)}-R_{i j}^{(0)}\right)+d .
$$

Proof. By the assumptions and the definition of $D_{\delta, \mu}$ in (1.5), we have

$$
\left|\beta^{(1)}-\beta^{(0)}\right|^{2} \leq n\left\|\beta^{(1)}-\beta^{(0)}\right\|^{2} \leq 2 n\left(\left\|\beta^{(0)}\right\|^{2}+\left\|\beta^{(1)}\right\|^{2}\right) \leq 4 n \mu^{2},
$$

and

$$
\delta \lambda_{\min }\left((1-s) \omega^{(0)}+s \omega^{(1)}\right) \geq \mu, \forall s \in[0,1] .
$$

From these estimates and (3.47), we can easily obtain the desired estimate (3.50).

\section{Comparison principle for nonsymmetric Monge-Ampère type} EQUATIONS

In this section, we shall establish the comparison principle for the MongeAmpère type equation (1.1) in the case that it is $\delta$-elliptic, $0 \leq \delta<1$ with respect to compared functions. Consider the following operator associated to the equation (1.1),

$$
G[u](x) \equiv \log \operatorname{det}\left[D^{2} u-A(x, u, D u)-B(x, u, D u)\right]-\log f(x, u, D u), x \in \Omega .
$$

Theorem 4. Let $u(x), v(x) \in C^{2}(\bar{\Omega})$ satisfying $G[u](x) \leq G[v](x)$ in $\Omega, u \geq v$ on $\partial \Omega$, where $A, B, f$ are in $C^{1}\left(\bar{\Omega} \times \mathbb{R} \times \mathbb{R}^{n}\right)$ and $f>0$ on $\bar{\Omega} \times \mathbb{R} \times \mathbb{R}^{n}$. Suppose that the following conditions are satisfied for some nonnegative constants $\delta, \alpha_{1}, \beta_{1}$, 
$0 \leq \delta<1$ and for all $x \in \bar{\Omega}, z \in \mathbb{R}, p \in \mathbb{R}^{n}$,

(i) $\lambda_{\min }(\omega(x, u))>0, \lambda_{\min }(\omega(x, v))>0$;

(ii) $\delta \min \left\{\lambda_{\min }(\omega(x, u)), \lambda_{\min }(\omega(x, v))\right\} \geq \mu(B)$;

(iii) $\lambda_{\min }\left(D_{z} A(x, z, p)\right) \geq\left(-\alpha_{1}\right) \min \left\{\lambda_{\min }(\omega(x, u)), \lambda_{\min }(\omega(x, v))\right\}$;

(iv) $\beta_{1} \min \left\{\lambda_{\min }(\omega(x, u)), \lambda_{\min }(\omega(x, v))\right\} \geq \mu\left(D_{z} B\right)$;

(v) $\inf _{\bar{\Omega} \times \mathbb{R} \times \mathbb{R}^{n}}\left(\frac{D_{z} f}{f}\right) \geq n\left(\alpha_{1}+\frac{\delta}{1+\delta^{2}} \beta_{1}\right)$,

where the quantities $\mu(B), \mu\left(D_{z} B\right)$ are defined as in (1.6).

Then we have that either $u>v$ or $u \equiv v$ in $\Omega$.

Proof. For all $x \in \bar{\Omega}$ and for all $t \in[0,1]$, we set

$$
\begin{gathered}
w(x)=v(x)-u(x), \\
u^{(t)}(x)=(1-t) u(x)+t v(x),
\end{gathered}
$$

and

$$
\begin{aligned}
& R^{(0)}(x)=D^{2} u(x)-A(x, u(x), D u(x))-B(x, u(x), D u(x)), \\
& R^{(1)}(x)=D^{2} v(x)-A(x, v(x), D v(x))-B(x, v(x), D v(x)), \\
& R^{(t)}(x)=(1-t) R^{(0)}(x)+t R^{(1)}(x), \\
& \omega^{(0)}(x)=D^{2} u(x)-A(x, u(x), D u(x)), \\
& \omega^{(1)}(x)=D^{2} v(x)-A(x, v(x), D v(x)), \\
& \omega^{(t)}(x)=(1-t) \omega^{(0)}(x)+t \omega^{(1)}(x) .
\end{aligned}
$$

Set

$$
g(t, x) \equiv \log \operatorname{det}\left((1-t) R^{(0)}(x)+t R^{(1)}(x)\right)=\log \operatorname{det}\left(R^{(t)}(x)\right) .
$$

Then by the mean value Theorem and (3.16), we have

$$
\begin{aligned}
\log \operatorname{det}\left(R^{(1)}(x)\right) & -\log \operatorname{det}\left(R^{(0)}(x)\right)=g(1, x)-g(0, x) \\
& =g_{t}^{\prime}(s, x)=\sum_{i, j=1}^{n}\left(R^{(s)}(x)\right)_{j i}^{-1}\left(R_{i j}^{(1)}(x)-R_{i j}^{(0)}(x)\right),
\end{aligned}
$$

where $s \in(0,1)$ is the constant depending on $x$.

Set

$$
\begin{aligned}
h(t, x)=\sum_{i, j=1}^{n} & \left(R^{(s)}(x)\right)_{j i}^{-1}\left[D_{i j} u^{(t)}(x)-A_{i j}\left(x, u^{(t)}(x), D u^{(t)}(x)\right)\right. \\
& \left.-B_{i j}\left(x, u^{(t)}(x), D u^{(t)}(x)\right)\right]-\log f\left(x, u^{(t)}(x), D u^{(t)}(x)\right) .
\end{aligned}
$$


Then by the mean value Theorem and (4.1), we obtain

$$
\begin{aligned}
& G[v](x)-G[u](x)=h(1, x)-h(0, x)=h_{t}^{\prime}(\tau, x) \\
& =\sum_{i, j=1}^{n}\left(R^{(s)}(x)\right)_{j i}^{-1}\left[\left(D_{i j} v(x)-D_{i j} u(x)\right)\right. \\
& -\sum_{k=1}^{n}\left(D_{p_{k}} A_{i j}+D_{p_{k}} B_{i j}\right)\left(x, u^{(\tau)}(x), D u^{(\tau)}(x)\right)\left(D_{k} v(x)-D_{k} u(x)\right) \\
& \left.-\left(D_{z} A_{i j}+D_{z} B_{i j}\right)\left(x, u^{(\tau)}(x), D u^{(\tau)}(x)\right)(v(x)-u(x))\right] \\
& -\frac{1}{f\left(x, u^{(\tau)}(x), D u^{(\tau)}(x)\right)} \sum_{k=1}^{n} D_{p_{k}} f\left(x, u^{(\tau)}(x), D u^{(\tau)}(x)\right)\left(D_{k} v(x)-D_{k} u(x)\right) \\
& -\frac{1}{f\left(x, u^{(\tau)}(x), D u^{(\tau)}(x)\right)} D_{z} f\left(x, u^{(\tau)}(x), D u^{(\tau)}(x)\right)(v(x)-u(x)),
\end{aligned}
$$

where $\tau \in(0,1)$ is the constant depending on $x$ and $s$.

Consequently,

$$
G[v](x)-G[u](x)=a^{i j}(x) D_{i j} w(x)+b^{k}(x) D_{k} w(x)+c(x) w(x),
$$

where

$$
\begin{aligned}
a^{i j}(x) & =\frac{\left(R^{(s)}(x)\right)_{i j}^{-1}+\left(R^{(s)}(x)\right)_{j i}^{-1}}{2} \\
b^{k}(x) & =-\sum_{i, j=1}^{n}\left(R^{(s)}(x)\right)_{j i}^{-1}\left[\left(D_{p_{k}} A_{i j}+D_{p_{k}} B_{i j}\right)\left(x, u^{(\tau)}(x), D u^{(\tau)}(x)\right)\right] \\
& -\frac{1}{f\left(x, u^{(\tau)}(x), D u^{(\tau)}(x)\right)} D_{p_{k}} f\left(x, u^{(\tau)}(x), D u^{(\tau)}(x)\right), \\
c(x) & =-\sum_{i, j=1}^{n}\left(R^{(s)}(x)\right)_{j i}^{-1}\left[\left(D_{z} A_{i j}+D_{z} B_{i j}\right)\left(x, u^{(\tau)}(x), D u^{(\tau)}(x)\right)\right] \\
& -\frac{1}{f\left(x, u^{(\tau)}(x), D u^{(\tau)}(x)\right)} D_{z} f\left(x, u^{(\tau)}(x), D u^{(\tau)}(x)\right) .
\end{aligned}
$$

Consider the second order linear partial differential operator $L$ given by

$$
L:=a^{i j}(x) D_{i j}+b^{k}(x) D_{k}+c(x),
$$

where the coefficients $a^{i j}, b^{k}, c$ are defined by (4.3). We have the following claims.

Claim 1. The operator $L$ is uniformly elliptic; that is, there exists positive constants $\lambda, \Lambda$ such that

$$
\lambda|\xi|^{2} \leq a^{i j}(x) \xi_{i} \xi_{i} \leq \Lambda|\xi|^{2}, \forall x \in \bar{\Omega}, \forall \xi \in \mathbb{R}^{n} .
$$

Indeed, it follows from conditions (i), (ii) that $R^{(0)}(x), R^{(1)}(x)$ are in the set $D_{\delta, \mu(B)}$, so is $R^{(s)}(x)$. Also from (i) and our regularity assumptions for $A, B$ and 
$u$, we infer that there exists positive constants $\lambda_{0}, \Lambda_{0}$ such that

$$
\lambda_{0} E \leq \omega(x, u) \leq \Lambda_{0} E, \quad \lambda_{0} E \leq \omega(x, v) \leq \Lambda_{0} E, \forall x \in \bar{\Omega},
$$

where $E$ is the unit matrix of order $n$. It follows that

$$
\lambda_{0} E \leq \omega^{(s)}(x) \leq \Lambda_{0} E, \forall x \in \bar{\Omega} .
$$

Therefore

$$
\frac{1}{\Lambda_{0}} E \leq\left(\omega^{(s)}(x)\right)^{-1} \leq \frac{1}{\lambda_{0}} E, \forall x \in \bar{\Omega} .
$$

Moreover, by Proposition 4 and Corollary 1, one can easily show that

$$
\frac{1}{1+\delta^{2}}\left(\left(\omega^{(s)}(x)\right)^{-1} \xi, \xi\right) \leq(H(x) \xi, \xi) \leq\left(\left(\omega^{(s)}(x)\right)^{-1} \xi, \xi\right), \forall x \in \bar{\Omega},
$$

where

$$
H(x):=\frac{\left(R^{(s)}(x)\right)^{-1}+\left(\left(R^{(s)}(x)\right)^{-1}\right)^{T}}{2} .
$$

Then (4.5) follows from the above estimates by taking $\lambda=\frac{1}{\left(1+\delta^{2}\right) \Lambda_{0}}$ and $\Lambda=$ $\frac{1}{\lambda_{0}}$.

Claim 2. The coefficients $b^{k}(x), c(x)$ are bounded in $\bar{\Omega}$.

This claim easily follows from the fact that the set $\left\{\left(x, u^{(\tau)}(x), D u^{(\tau)}(x)\right)\right\}$ is bounded in $\bar{\Omega} \times \mathbb{R} \times \mathbb{R}^{n}$ and

$$
\operatorname{det} R^{(s)}(x) \geq \operatorname{det} \omega^{(s)}(x) \geq \lambda_{0}^{n}>0, \forall x \in \bar{\Omega},
$$

which holds by Proposition 3 and (4.6).

Claim 3. The coefficient $c(x) \leq 0$ for all $x \in \bar{\Omega}$.

Claim 3 follows from (4.3), condition (v) and the two following inequalities

$$
\begin{gathered}
-\sum_{i, j=1}^{n}\left(R^{(s)}(x)\right)_{j i}^{-1} D_{z} A_{i j}\left(x, u^{(\tau)}(x), D u^{(\tau)}(x)\right) \leq n \alpha_{1}, \forall x \in \bar{\Omega}, \\
-\sum_{i, j=1}^{n}\left(R^{(s)}(x)\right)_{j i}^{-1} D_{z} B_{i j}\left(x, u^{(\tau)}(x), D u^{(\tau)}(x)\right) \leq n \frac{\delta}{1+\delta^{2}} \beta_{1}, \forall x \in \bar{\Omega} .
\end{gathered}
$$

So it remains to prove (4.7) and (4.8).

Since $D_{z} A$ is symmetric and $H(x)$ is positive definite, we have

$$
\sum_{i, j=1}^{n}\left(R^{(s)}(x)\right)_{j i}^{-1} D_{z} A_{i j}=\operatorname{Tr}\left[\left(D_{z} A\right)(H(x))\right] \geq \lambda_{\min }\left(D_{z} A\right) \operatorname{Tr} H(x) .
$$

Given any point $x \in \bar{\Omega}$. Assume that $\lambda_{\min }\left(D_{z} A\left(x, u^{(\tau)}(x), D u^{(\tau)}(x)\right)\right) \geq 0$ at this point. Then by (4.9), the left hand side of (4.7) is nonpositive and thus (4.7) 
follows. Assume the contrary, that $\lambda_{\min }\left(D_{z} A\left(x, u^{(\tau)}(x), D u^{(\tau)}(x)\right)\right)<0$. Then (4.7) follows from (4.9) and the following estimates

$$
\begin{aligned}
\operatorname{Tr} H(x) \leq & \operatorname{Tr}\left[\left(\omega^{(s)}(x)\right)^{-1}\right] \leq \frac{n}{\lambda_{\min }\left(\omega^{(s)}(x)\right)}, \\
\left(-\alpha_{1}\right) \lambda_{\min }\left(\omega^{(s)}(x)\right) & \leq\left(-\alpha_{1}\right) \min \left\{\lambda_{\min }(\omega(x, u)), \lambda_{\min }(\omega(x, v))\right\} \\
& \leq \lambda_{\min }\left(D_{z} A\left(x, u^{(\tau)}(x), D u^{(\tau)}(x)\right)\right),
\end{aligned}
$$

which are inferred from Corollary 2 and condition (iii), respectively. Thus (4.7) is proved.

We now prove (4.8). Set

$$
K(x):=\frac{\left(R^{(s)}(x)\right)^{-1}-\left(\left(R^{(s)}(x)\right)^{-1}\right)^{T}}{2} .
$$

By Proposition 4 and Corollary 1, one can easily show that

$$
\|K(x)\| \leq \frac{\delta}{1+\delta^{2}} \frac{1}{\lambda_{\min }\left(\omega^{(s)}(x)\right)}, \forall x \in \bar{\Omega},
$$

and, by condition (iv),

$$
\mu\left(D_{z} B\right) \leq \beta_{1} \min \left\{\lambda_{\min }(\omega(x, u)), \lambda_{\min }(\omega(x, v))\right\} \leq \beta_{1} \lambda_{\min }\left(\omega^{(s)}(x)\right), \forall x \in \bar{\Omega} .
$$

From these estimates and the following inequality:

$$
\operatorname{Tr}(M N) \leq|M||N| \leq n\|M\|\|N\|, \text { for all } M, N \in \mathbb{R}^{n \times n},
$$

we obtain for all $x \in \bar{\Omega}$,

$$
\begin{aligned}
-\sum_{i, j=1}^{n}\left(R^{(s)}(x)\right)_{j i}^{-1} & D_{z} B_{i j}\left(x, u^{(\tau)}(x), D u^{(\tau)}(x)\right) \\
& =\operatorname{Tr}\left[(K(x))\left(-D_{z} B\left(x, u^{(\tau)}(x), D u^{(\tau)}(x)\right)\right)\right] \\
& \leq n \frac{\delta}{1+\delta^{2}} \frac{\mu\left(D_{z} B\right)}{\lambda_{\min }\left(\omega^{(s)}(x)\right)} \leq n \frac{\delta}{1+\delta^{2}} \beta_{1} .
\end{aligned}
$$

Thus (4.8) is proved.

To complete the proof of this theorem, we note that, if $G[u] \leq G[v]$ in $\Omega, u \geq$ $v$ on $\partial \Omega$ then, by (4.2) and (4.4), $L w \geq 0$ in $\Omega, w \leq 0$ on $\partial \Omega$. By Claims 1, 2, 3, we can apply the strong maximum principle of E. Hopf (Theorem 3.5, 2] ) to obtain the conclusion of Theorem 4 .

Corollary 5. Under the assumptions of Theorem 4, where $G[u]<G[v]$ in $\Omega$, $u=v$ on $\partial \Omega, \partial \Omega \in C^{2}$, we have the following strict inequalities

$$
\begin{gathered}
u>v, \text { in } \Omega, \\
\frac{\partial u}{\partial \nu}>\frac{\partial v}{\partial \nu}, \text { on } \partial \Omega,
\end{gathered}
$$


where $\nu$ is the unit inner normal to $\partial \Omega$.

Proof. Set $w=v-u$. Following the proof of Theorem 4, we have

$$
L w>0 \text { in } \Omega, w=0 \text { on } \partial \Omega,
$$

where $L$ is the uniformly elliptic operator defined by (4.4). Further, by Theorem 4. we have that $w<0$ in $\Omega$. Hence, we can apply the Hopf's lemma (Theorem $3.4,[2]$ ) to obtain

$$
\frac{\partial w}{\partial \nu}<0, \text { on } \partial \Omega
$$

The proof is completed.

\section{REFERENCES}

[1] Aitken, A.C., Determinants and matrices, Oliver and Boyd, Edinburgh, 1956.

[2] Gilbarg, D., Trudinger, N.S., Elliptic partial differential equations of second order, SpringerVerlag, Berlin-New York, 2001.

[3] Ha Tien Ngoan, Thai Thi Kim Chung, Elliptic solutions to nonsymmetric Monge-Ampère type equations II. A priori estimates and the Dirichlet problem (in preparation).

[4] Jiang, F., Trudinger, N.S., Yang, X.-P., On the Dirichlet problem for Monge-Ampère type equations, Calc. Var. PDE., 49 (2014), 1223-1236.

[5] Jiang, F., Trudinger, N.S., Yang, X.-P., On the Dirichlet problem for a class of augmented Hessian equations, J. Diff. Eqns., 258 (2015), 1548-1576.

[6] Trudinger, N.S., Recent developments in elliptic partial differential equations of MongeAmpère type, Proc. Int. Cong. Math., Madrid, 3 (2006), 291-302.

Institute of Mathematics, Vietnam Academy of Science and Technology, 18 Hoang Quoc Viet, 10307 Cau Giay, Hanoi, Vietnam.

E-mail address: htngoan@math.ac.vn

University of Transport Technology, 54 Trieu Khuc, Thanh Xuan, Hanoi, VietNAM

E-mail address: chungttk@utt.edu.vn 